\title{
The Role of Average Health Status - Health Inequalities Matrix for Assessing Impacts on Population Health in Health in All Policies
}

Jordan Panayotov, Independent Centre for Analysis and Research of Economies (ICARE), Balwyn, Australia

Please send correspondence to:

Jordan Panayotov, Independent Centre for Analysis and Research of Economies

PO Box 437, Balwyn VIC 3103, Australia

E-mail: jp@icare.biz

\begin{abstract}
Economic, social and environmental policies, programs and projects have impacton health. Health in All Policies (HiAP) aims to improve population health by taking into account these impacts. HiAP needs appropriate tools for assessing impacts on population health. When making choices between policy options, decision-makers rely on predictions from Health Impact Assessment. Currently there is no gold standard for establishing and assessing validity of predictions. This paper distinguishes between two levels of causal pathways regarding health impacts - specific and conditional, and proposes the Average Health Status - Health Inequalities Matrix as gold standard. The Matrix facilitates making the right choices at any level and local context, thus is useful for researchers, policy-makers and practitioners for designing, analysing and evaluating all kinds of policies. By allowing quick, reliable and inexpensive appraisal of different policy options the matrix makes feasible taking into account the impacts on population health and paves the way for institutionalizing of HiAP.
\end{abstract}

Keywords: $\quad$ Evidence, Health in All Policies, Impact Assessment, Population Health, Scenarios for Policy Options, Validity of Predictions

\section{INTRODUCTION}

\section{Simplicity is the ultimate sophistication (Leonardo da Vinci)}

There is increasing awareness that policies in all sectors have some impact on health. Health in All Policies approach aims to improve population health and health equity by taking into account these impacts when decisions are made (WHO, 2014). In order to be successful, apart from political will and resources, Health in All Policies needs appropriate tools for proper assessment 
of impacts on population health from different policy options. Such tools include: Social Impact Assessment which looks at social consequences from a policy and aims sustainable development (Becker, 1997); Health Impact Assessment (HIA) which explicitly looks for and examines impacts on population health (Lehto \& Ritsatakis, 1999); Average Health Status - Health Inequalities Matrix (Panayotov 2006b; 2008a) which looks at the impacts on population health from different policy options in terms of changes in average health status and health inequalities; and EquityFocussed Evaluation - an emerging concept with developing methodology, which is concerned with achieving equitable development (Bamberger \& Segone, 2011).

Melkas (2013) suggests that "HIA is a key tool". However, Panayotov (2010) points out that there are problems with HIA methodology, and policies can create and/or widen health inequalities, even if nobody is worse-off (see Examples, Case I). How to determine which is the best tool for successful Health in All Policies approach?

Population health has two major variables: average health status (AHS) and health inequalities (HI). During the last six decades decision-makers around the world were focused on improving AHS. HI are discussed since 1990 and Douglas \& Scott-Samuel (2001) suggest that HIA should address HI, recognising that there is tension arising from the trade-offs between improving AHS and improving health of the most disadvantaged. What should decision-makers strive for in relation to population health: improving $A H S$, or reducing $H I$ ? While analysing the correlation between these two variables, Panayotov (2006a) points out that although that these two goals may look similar, they have different paths which lead to different ends. For example, AHS will improve, when the health of those who are better-off improves faster than rest of the population, but as result HI will increase. He points out that from an economics point of view creating and/ or widening of HI represents inefficient and unsustainable allocation of limited public resources (Panayotov, 2006b). Showing that in relation to population health achieving equity is a premise for efficiency (maximizing population health with limited available resources), Panayotov (2006b) asserts that HI should be reduced not only on compassionate grounds. Therefore, he suggests that the goal for improving AHS should go hand-in-hand with the goal for reducing HI.

Since neglecting health will cost much more later, the negative, mainly direct impacts are usually addressed and mitigated, often required by the local legislation. Vanclay et al. (2015) note that the focus of Social Impact Assessment has shifted from primarily addressing the negative impacts to enhancing the benefits (i.e. positive impacts). However, it has been noted that while enhancing positive impacts is a prima facie improvement, it can create and/or widen HI (Panayotov 2008a; 2008b; 2010). This happens when people who benefit more from the new policy are those who already are better-off. Panayotov $(2008 \mathrm{a} ; 2008 \mathrm{~b} ; 2010)$ asserts that for policies distribution of the benefit among the population is an important factor influencing outcomes, whether improving health is the primary objective (health policies, see Examples, Case I and Case IV) or the primary objective is different than health (policies in other sectors: energy, transport, education, agriculture, urban planning, etc., see Examples, Case II and Case III). In other words, the distribution of the benefit from different policy options determines population health and/ or its determinants. Panayotov (2008a; 2008b) concludes that achieving equitable development (what Social Impact Assessments and Equity-Focussed Evaluations are about) is a premise for reducing HI and improving population health (what Health in All Policies and HIA are about).

By definition HIA is concerned with the potential and/or unintended effects on population health and the distribution of those effects within the population (Lehto \& Ritsatakis, 1999). However, Kemm (2006) notes that many HIAs often "fall short of a proper analysis of distribution of impacts detailing how the various impacts would fall on different groups within the population". Probably because of this, the Commission on Social Determinants of Health (Marmot et al., 2008) has recommended assessment of health equity effects of public policy decisions by

Copyright (C) 2015 The Author. This article published as an Open Access article distributed under the terms of the Creative Commons Attribution NonCommercial 4.0 License (http://creativecommons.org/licenses/by-nc/4.0/). 
conducting health equity impact assessment (Recommendations:10.3;12.1;16.7). Panayotov (2011) points out that there is no need to invent a new name in order to conduct proper analysis of impacts' distribution among the population and to make recommendations for improving health equity. First, putting out new names diminishes the value and credibility of HIA. Second, the use of "health equity impact assessment" or "equity focused health impact assessment" implies that there can be 'other' types of HIA which do not specifically consider equity, and this undermines the whole idea for addressing and reducing HI, since these can be ignored in the 'other' HIAs. If properly conducted all HIAs should provide recommendations to improve health equity.

When making choices, decision-makers rely on the predictions from HIA. However, currently HIA has no gold standard - a test which always identifies the true condition - for establishing and assessing validity of predictions. This paper proposes that the Average Health Status - Health Inequalities Matrix (AHS-HI Matrix) could be such standard. This is a model for assessment of policies, programs, projects and other interventions on populations (hereafter the term policy will be used for all of them) regarding their impact on population health in terms of changes in the two major variables: AHS and HI. This paper will regard the role of AHS-HI Matrix only with HIA, since HIAs, Social Impact Assessments and Equity-Focussed Evaluations have the same domain and objectives and overlap substantially.

\section{THE ROLE OF HIA FOR IMPROVING POPULATION HEALTH}

Any policy is a decision for resource allocation with winners - people who benefit from it, and losers - people who benefit less, not at all, or are worse-off compared to their previous situation. Panayotov (2008a) points out that when people benefit less from a policy, but they are not worse-off compared to their situation before the change, they are relative losers. People are absolute losers, if as a result from the change, they are worse-off compared to their previous situation. Further, he asserts that when people benefit less before the change and also less from the new policy, they are double losers and this can create and/or widen HI, even if nobody is an absolute loser.

It is important to note that HIA should not be confined to or confused with health risk assessment and/or risk management. Banken (1999) suggests that HIA should be more about prediction of tendencies and types of impacts rather than absolute measures. In agreement with Banken, this paper argues that decision-makers should be more concerned with answers regarding impacts on population health in terms of changes in AHS and HI. Therefore, in order to be useful for decision-makers HIA should:

1. Explain what will happen with AHS and HI for each of the identified options/alternatives with clear statements of the following type: "If you do option 1, 2, or 3, then you'll get (population health) outcome X, Y, or Z"; and

2. Explain why this will happen, i.e. on what basis the predictions are made.

Outcomes corresponding for each policy option should be clearly defined, just as alternative policy options are, taking into account changes in AHS and HI. This requires that the "what" part should be done not for the population on average, but for stratified population groups which should reflect specificities of the local context (Panayotov, 2009). HIA should explicitly state what stratification of the population is considered (this may vary from case to case) and what is the rationale behind it (why such differentiation and increments are chosen). When specific health outcomes might be uncertain, unclear or otherwise unknown (especially due to complex- 
ity), outcomes about the determinants of health should be used instead, pointing out their impact on AHS and HI.

Since populations have normal distribution with bell-shape, which is defined by two parameters: mean (average) $\mu$ and standard deviation $\sigma$, Panayotov (2009) points out that the goal for improving population health means that decision-makers should strive for such distribution of the benefit among the population, which leads to reducing the standard deviation (proxy for HI) while improving the mean (AHS). This means that the population bell should become narrower (reduced HI) while moving to the right (i.e. increased life expectancy, improved Quality-of-Life and well-being) or moving to the left (i.e. reduced exposure, morbidity, mortality). On the other hand, widening HI will flatten the population bell, even if AHS improves. If presented correctly, policy impacts are easy to see, compare and comprehend even by lay people (Panayotov, 2010).

Both decision-makers and HIA practitioners should be aware that since "In HIA prediction is based on a set of causal or logic models" (Kemm 2006), quality/validity of predictions depends on quality/validity of the model, which in turn depends on quality of the theoretical framework used. It should be noted that at present regarding policies there are problems with validity of both causal and logic models. For example, Kelly et al. (2006) note that in relation to HI it appears from the evidence that in many cases the causal pathways are not always so clear and the covering laws (by which authors obviously mean the generative mechanisms) are not known at all. These problems stem from the fact that those models do not consider distribution of the benefit from a policy/intervention among the population. Panayotov $(2008 \mathrm{~b} ; 2010)$ points out that for any specific case, no matter what the concrete causal pathways are (whether known and clear, or not), the effects in which HIA is interested in are always a consequence from distribution of the benefit from implemented policy/intervention among the population. AHS-HI Matrix is a model which shows how distribution of the benefit from a policy/intervention impacts population health in terms of changes in both AHS and HI. In order to be valid a model should be based on proper theoretical framework.

\section{THE ROLE OF THEORY}

Theoretical frameworks play fundamental role in constructing a model, since any flaw in them will inevitably affect negatively validity of the model. Decisions based on recommendations generated by problematic models may not be successful in achieving predetermined goal (for example, improving population health) and can be even counterproductive by creating and/or widening HI. Heller et al. (2004) stress that "unless public health programs are based on sound theoretical bases, they will fail". I want to emphasise that this applies also to recommendations in HIA.

In a treatise on scientific methodology in social sciences, Popper (1957) investigates the role of theory in testing a hypothesis, explaining observations and predicting outcomes. He points out that initial conditions influenced by the force of universal laws described in a theoretical framework result in outcomes. It is suggested that, like in mathematics, when two variables are known, the third can be inferred. For example, when the theoretical framework is valid and outcomes are known, initial conditions can be inferred. Similarly, when the theoretical framework is valid and initial conditions are known (the status quo which decision-makers want to change and different policy options to do this), outcomes corresponding to each option can be inferred (i.e. prediction can be made). Popper asserts that, when initial conditions cannot be explained or when outcomes differ from the predictions, the theoretical framework is falsified or refuted. $\mathrm{He}$ concludes that theoretical frameworks which better "survive" the process of refutation are more 
applicable to problem situations in real life. For appraisal of theories Popper states that theoretical frameworks can be judged objectively by the amount of truth that they imply. Thus, theoretical frameworks based on universal laws would provide more truth, as these will be valid in all cases.

Connelly $(2001 ; 2005 ; 2007)$ notes that only theoretical frameworks based on critical realism (if $\boldsymbol{A}$ then always $\boldsymbol{B}$ ) can provide universal explanations and predictions. Theoretical frameworks inferred only from empirical observations of outcomes and initial conditions might not be able to provide universal explanations and valid predictions. Validity may be problematic, if the universal laws (generative mechanisms) are not known. Apparently, this is the case regarding HI, because in spite of the abundance of empirical observations of outcomes and initial conditions, the Commission on Social Determinants of Health could not identify the universal laws (generative mechanisms) which create, widen, or diminish HI, and consequently there are no universal explanations and predictions provided in its final Report (Marmot et al. 2008). Not surprisingly Venkatapuram (2010), although praising the CSDH's Final Report as a positive step for addressing health in human development, notes that "the commission's policy recommendations are quite general" and admits that there are multiple problems with implementing the Commission's recommendations at local level in different countries. By revealing the universal laws (generative mechanisms) about impacts on population health from different policy options AHS-HI Matrix provides universal explanations and predictions. Thus it can be used as a gold standard facilitating making the right choices for addressing social determinants of health in order to reduce HI and improve population health at any local context.

\section{THE ROLE OF AVERAGE HEALTH STATUS - HEALTH INEQUALITIES MATRIX}

Matrices are known as a useful tool for demonstrating correlation between variables. Thérivel et al. (1992) point out their usefulness for identifying different impacts. If properly constructed, with all considered alternatives on one axis and the corresponding impacts on the other axis, a matrix can be very useful for comparing different options (Becker 1997), because the impacts are presented in a clear and systematic way (Barrow 2000). Kauppinen et al. (2006) note that regarding health "the same matrix can simultaneously deal with impacts, determinants of health or objectives" and point out that one main purpose of a matrix is to show the positive and negative impacts of different options on HI. However, regarding health impacts from policies, it has been noted that there are many uncertainties about causal pathways (Kelly et al. 2006; Kemm 2006; Mindell et al. 2004). Consequently, these uncertainties present considerable challenges when constructing a matrix. However, as already mentioned, no matter what the concrete causal pathways are, impacts on population health are always a consequence from distribution of the benefit from a policy among the population. According to Panayotov (2008a) this distribution is an important factor influencing the outcomes which in turn determine the impacts on population health manifested as changes in AHS and HI.

From any policy/intervention people can be either better-off, worse-off, or without a change (i.e. impacts can be either positive, negative, or neutral). On the vertical axis are all (nine) theoretically possible combinations of previous and new winners and losers from any policy/intervention on populations. "Yes" indicates change, and any row can have maximum of two times "Yes", as it is not possible for one to be simultaneously better-off and worse-off. On the horizontal axis are the corresponding impacts on population health in terms of changes in AHS and HI, both of which can increase, decrease or remain the same. It is assumed that it is not possible for one to be better-off from an intervention and this to be bad for one's health, and respectively it is not 
possible for one to be worse-off from an intervention and this to be good for one's health. Thus, any specific combination of previous and new winners and losers can achieve nothing else, but the corresponding impact on AHS and HI. Therefore, being based on critical realism - if $\boldsymbol{A}$ (specific combination of previous and new winners and losers) then always $\boldsymbol{B}$ (corresponding for this combination impact on AHS and $\mathrm{HI}$ ) the matrix provides universal explanations and predictions for impacts on population health from different policy options (see Table 1).

AHS-HI Matrix has several benefits when used as a tool for analysis of health impacts from policies, projects, programs or other interventions on populations:

- Universal applicability: Since all possible combinations of distribution of the benefit from a policy or intervention on populations are considered, the matrix is applicable for assessment of any policy or intervention anywhere in the world, since no matter how different the local context is, there always will be winners and losers from this policy;

- Comprehensiveness: All possible options (alternatives) of distribution of the benefit from a policy or intervention are considered in the matrix;

- Simplicity: The matrix provides simple descriptions, yet sufficient for purposes of informing decision-makers;

- Clarity: All possible options together with their corresponding impacts are presented in an easy to see, comprehend and compare, clear and systematic way;

Table 1. Average Health Status - Health Inequalities Matrix. (Copyright (C) 2008 ICARE. All rights reserved. Reproduced by permission of Independent Centre for Analysis \& Research of Economies from: Panayotov J., 2008, Public Health and Average Health Status: Do Health Inequalities Matter?, ICARE, 08 August 2008: Table 2)

\begin{tabular}{|c|c|c|c|c|c|c|c|c|c|c|}
\hline \multicolumn{10}{|c|}{ Outcome from New Policy, Program or Intervention } & \multirow[b]{3}{*}{$\begin{array}{c}\text { Case } \\
\text { (Scenario) }\end{array}$} \\
\hline \multicolumn{2}{|c|}{ Better-Off } & \multicolumn{2}{|c|}{ Worse-Off } & \multicolumn{3}{|c|}{ A H S } & \multicolumn{3}{|c|}{ H I } & \\
\hline $\begin{array}{l}\text { Previous } \\
\text { Winners }\end{array}$ & $\begin{array}{c}\text { Previous } \\
\text { Losers }\end{array}$ & $\begin{array}{l}\text { Previous } \\
\text { Winners }\end{array}$ & $\begin{array}{c}\text { Previous } \\
\text { Losers }\end{array}$ & $\uparrow$ & $\downarrow$ & - & $\uparrow$ & $\downarrow$ & - & \\
\hline YES & NO & $\mathrm{NO}$ & NO & $\mathrm{X}$ & & & $\mathrm{X}$ & & & 1 \\
\hline NO & YES & NO & NO & $\mathrm{X}$ & & & & $\mathrm{X}$ & & 2 \\
\hline NO & NO & NO & YES & & $\mathrm{x}$ & & $\mathrm{X}$ & & & 3 \\
\hline NO & NO & YES & NO & & & & & $\mathrm{X}$ & & 4 \\
\hline YES & NO & NO & YES & $x^{*}$ & $x^{*}$ & $\mathrm{x}^{*}$ & $\mathrm{X}$ & & & $1 *, 3 *, 5$ \\
\hline $\mathrm{NO}$ & YES & YES & NO & $\mathrm{x}^{*}$ & $x^{*}$ & $\mathrm{x}^{*}$ & & $\mathrm{X}$ & & $2 *, 4 *, 6$ \\
\hline YES & YES & NO & NO & $\mathrm{X}$ & & & $\mathrm{x}^{\wedge}$ & $\mathrm{x}^{\wedge}$ & $\mathrm{x}^{\wedge}$ & $1^{\wedge}, 2^{\wedge}, 7$ \\
\hline NO & NO & YES & YES & & $\mathrm{X}$ & & $x^{\wedge}$ & $x^{\wedge}$ & $\mathrm{x}^{\wedge}$ & $3^{\wedge}, 4^{\wedge}, 8$ \\
\hline NO & NO & $\mathrm{NO}$ & NO & & & $X$ & & & $\mathrm{X}$ & 9 \\
\hline
\end{tabular}

AHS - average health status

HI - health inequalities

* Whether AHS increases, decreases or remains the same depends on the balance of the gain/loss between recipients (can be positive, negative, or neutral).

$\wedge$ Whether HI increase, decrease or remain the same depends on the balance of the gain/loss between recipients (can be positive, negative, or neutral). 
- Uncompromised validity: Since all possible combinations of distribution of the benefit are considered and each one of these comes with only one corresponding outcome, all predictions that are made based on the matrix have the highest possible validity;

- Efficiency: The matrix requires significantly less time and resources: (i) for proper analysis of various impacts from all options/alternatives and their distribution within the population; and (ii) for collating and appraisal of relevant evidence;

- Versatility: The matrix facilitates swift adaptation to possible changes in objectives, available options/alternatives and/or the local context;

- Fairness: The matrix always shows the right way to reduce HI;

- Flexibility: The matrix can be tailored to diverse local conditions;

- Reproducibility: The matrix shows precisely what distribution of the benefit at local level is needed in order to achieve the desired outcome regarding the impact on AHS and $\mathrm{HI}$ in any specific case.

When choosing between different policy options decision-makers need tools which have the ability to:

1. Be applied across sectors and disciplines;

2. Develop and assess different scenarios, often in changing conditions;

3. Deal with complexity and uncertainties;

4. Be feasible, i.e. simple and easy use, allowing timely and inexpensive appraisal of the available options.

By meeting all these requirements, AHS-HI Matrix can be a successful such tool.

\section{ENHANCING VALIDITY FOR POLICIES AND INTERVENTIONS ON POPULATIONS}

HIA makes sense for decision-makers only if the predictions that it makes are valid. Veerman et al. (2007) examine the relevance of different forms of validity to HIA, pointing out that at present "it is unclear how the validity of predictions should be defined in HIA, and how it can be assessed". They suggest that only "three types of validity are relevant for HIA: plausibility, formal validity and predictive validity" and HIA studies and methods should be assessed for plausibility and formal validity only, since there are no gold standards to establish the predictive validity. While in agreement with Veerman et al. regarding usefulness of Popper's work (1957) for establishing and assessing validity of predictions in HIA, we have disagreement on some of their conclusions regarding the relevance of different forms of validity to HIA. Below is shown the usefulness of AHS-HI Matrix for different forms of validity in HIA, including its usefulness as a gold standard for establishing and assessing validity of predictions, plus a new form of validity - validity of evidence - is introduced.

According to Popper (1957) identical interventions applied to identical conditions lead to identical outcomes. Then, validity of predictions about the outcomes can be:

- Established by confirming that initial conditions which will be modified and the policy/ intervention which will be applied do conform to the ones already held to be valid to a gold standard; and

- Assessed by confirming that the outcomes achieved are identical with the ones from the gold standard. 
In order to establish validity of predictions in HIA the internal validity should be in order in first place - that is the degree to which conclusions about causal relationships are valid. HIA relies on other disciplines to establish the specific causal pathways between health outcomes and exposure, or risk factors, or determinants of health. Veerman et al. (2007) assume these causal pathways to be valid and note that "HIA itself is not primarily intended to investigate causal relationships". Therefore they conclude that "the concept of internal validity does not directly apply to HIA". However, their assumption is problematic, since it is noted by many (Kelly et al. 2006; Kemm 2006; Mindell et al. 2004) that regarding social determinants of health (which are shaped by policies and/or interventions on populations) the causal pathways are not always as clear as they are for biological interventions (which are interventions on individuals). Mindell et al. (2004) point out that HIA is concerned with how different determinants of health are affected by the changes resulting from the proposed policy/intervention, however, still no clarification on the causal pathways is offered.

This paper distinguishes between two different types (levels) of causal pathways which are often confused, although they should not be. These types (levels) are to be called specific (level one) and conditional (level two).The relationship between these two is that specific causal pathways (what epidemiology is interested in) are affected by the conditional causal pathways (the distribution of the benefit from a policy among the population) as result of implemented policies. Conditional causal pathways will determine the extent to which the specific causal pathways will manifest in specific local context. Therefore, a specific causal pathway can lead to different outcomes due to different distribution of the benefit (i.e. conditional causal pathways). For example, population's exposure to noise and air pollution (well-established specific causal pathways) is affected by: (i) location of the highway; and (ii) interventions to mitigate the noise and air pollution (for example, using noise barriers, high-volume low-speed fans and air vents/ shafts, traffic regulations/restrictions, etc.); both of which are determined from the decisions made by decision-makers (conditional causal pathways). In other words, conditional causal pathways (level two) determine the internal validity for policies/interventions on populations. Therefore, HIA should be concerned with establishing the conditional causal pathways which will determine the changes in the determinants of health. Thus, regarding conditional causal pathways the concept of internal validity applies to HIA. For HIA it is of paramount importance to get the internal validity right in first place, in order to get any predictions right.

As mentioned above, AHS-HI Matrix always explains when conclusions about the conditional causal pathways regarding changes in both AHS and HI are valid. A specific combination of previous and new winners and losers (row in the matrix) leads to the precise and the only corresponding outcome regarding changes in AHS and HI. Different combinations of previous and new winners and losers will lead to different manifestations of the same specific causal pathways (whether these are known and well-established or not), thereby achieving different outcomes. If the outcome regarding changes in AHS and HI from specific combination of previous and new winners and losers is not satisfying (i.e. it deviates from the intended), AHS-HI Matrix shows what should be changed in order to achieve the desired outcome. In other words, the matrix identifies what change in the distribution of the benefit from the policy/intervention is needed (on matrix' left-hand side) in order to achieve the desired outcome for AHS and HI (on matrix' right-hand side). These nine combinations are the conditional causal pathways for policies/interventions on populations and in every specific case AHS-HI Matrix allows us to determine whether the conclusions about these causal relationships are valid, i.e. to establish the internal validity in HIA.

External validity represents the extent to which internally valid results can be held to be valid in other cases. However, it is important to point out that identical policies or interventions will

Copyright (C) 2015 The Author. This article published as an Open Access article distributed under the terms of the Creative Commons Attribution NonCommercial 4.0 License (http://creativecommons.org/licenses/by-nc/4.0/). 
achieve different outcomes regarding AHS and HI when there is a difference in the conditional causal pathways (i.e. in distribution of the benefit at local level). Replicating identical policy or intervention will achieve different outcomes regarding changes in AHS and HI in all cases where there is a different combination between previous and new winners and losers from this policy/intervention. On the other hand, the same outcomes regarding changes in AHS and HI can be achieved only by policy option with the corresponding for this outcome combination of previous and new winners and losers. This means that conditional causal pathways determine the external validity. AHS-HI Matrix helps to establish the external validity by showing whether identical policy/intervention will deliver the same outcome in other cases. This will happen only if the conditional causal pathways are externally valid, i.e. when the specific combination between previous and new winners and losers (row in the matrix) in other cases is the same as the one from the original case.

Representation validity represents the extent to which a theoretical framework can be operationalised (i.e. used in diverse real world). AHS-HI Matrix can be used in any local context and at any level of decision-making, since, no matter how different a specific case can be, there always will be winners and losers from a policy, which in turn will affect the outcome for both AHS and HI. Thus, AHS-HI Matrix has high representation validity.

Face validity indicates whether the theoretical framework used is appropriate and plausible to measure what it intends to measure. AHS-HI Matrix is appropriate and plausible to measure changes in AHS and HI, not only because it accounts for whether both of them are affected by a policy, but also because it considers all potential alternatives/options (i.e. possible combinations between previous and new winners and losers from this policy) which can affect the outcome for AHS and HI. Thus, AHS-HI Matrix has high face validity.

Criterion validity represents the extent to which the available information about a set of variables can predict the outcome for another variable. In relation to population health, the information about a set of variables consisting of previous and new winners and losers can predict changes in the other variables: AHS and HI. It is widely recognised that the best way to establish validity of predictions about the outcome is comparison to a gold standard - a test which always identifies the true condition. Veerman et al. (2007) note that at present for HIA "there are no such standards" and conclude that "we have to make do with less than the gold standard". AHS-HI Matrix can be used as a gold standard, since it always shows the true condition about the outcome (changes in AHS and HI) from a set of variables (previous and new winners and losers). Thus, AHS-HI Matrix has high criterion validity.

Predictive validity represents the extent to which predictions about future or independent past events become a reality. Since AHS-HI Matrix always shows the true condition about the outcome (changes in AHS and HI), any prediction regarding changes in these outcomes based on the matrix will be correct and will materialize. Thus, AHS-HI Matrix has high predictive validity.

Construct validity is about the appropriateness of the scale in order to operationalise a theoretical framework. AHS-HI Matrix has the appropriate scale to deliver predictions about "tendencies and types of impacts" regarding AHS and HI, rather than absolute measures about individuals, precisely what the decision-makers need. Thus, AHS-HI Matrix has high construct validity.

Content validity is about the extent to which a measure represents all facets of a given social construct. AHS-HI Matrix represents simultaneously the set of variables (previous and new winners and losers) and the outcome (changes in AHS and HI) for a given social construct (policies). Thus, AHS-HI Matrix has high content validity.

Convergent validity represents the extent to which the assessment is related to what it should theoretically be related to. Assessments provided on the basis of AHS-HI Matrix are entirely related to what they theoretically should be related to - the changes in AHS and HI as 
result from different choices of winners and losers from a policy. Thus, AHS-HI Matrix has high convergent validity.

Discriminant validity represents the extent to which the scale used to operationalise a theoretical framework differs from other scales designed to measure different theoretical constructs. The scale of AHS-HI Matrix which is intended to measure changes in population health (i.e. changes in AHS and HI) is completely different from the scales designed to measure the health of individuals, for example. Thus, AHS-HI Matrix has high discriminant validity.

Logical validity (also known as formal validity) is about whether the conclusion logically follows from the premises which are considered to be true. Conclusions made on the basis of AHS-HI Matrix logically follow from the premise that different distributions of the benefit from a policy/intervention (i.e. different combinations between previous and new winners and losers) lead to different outcomes regarding AHS and HI. Thus, AHS-HI Matrix has high logical validity.

Based on Occam's razor and Chatton's anti-razor principles a new, dialectic type of validity for establishing validity of evidence is suggested here.

Occam's razor validity represents the extent to which the evidence is most likely to be correct. It is based on Occam's razor principle that the simplest comprehensive description (of the evidence) is most likely to be correct. Vitanyi \& $\mathrm{Li}$ (2000) have proven a mathematical criterion for evaluation of evidence, which confirms the validity of Occam's razor principle. For policies AHS-HI Matrix allows the simplest, yet comprehensive description of the evidence regarding changes in AHS and HI. On this basis, any evidence and conclusions inferred by AHS-HI Matrix will have high likelihood to be correct.

Chatton's anti-razor validity represents the extent to which the evidence is likely to be incorrect, because a simple description of the things might not be sufficient to verify an affirmative proposition. Formally it states that "If three things are not enough to verify an affirmative proposition about things, a fourth must be added, and so on". In other words, the evidence is most likely to be correct if Chatton's anti-razor validity is low. AHS-HI Matrix does not need to add more things in order to verify an affirmative proposition regarding changes in AHS and HI from a policy. Therefore, any evidence and conclusions inferred by AHS-HI Matrix will have low likelihood to be incorrect.

AHS-HI Matrix provides high validity of evidence regarding changes in AHS and HI from policies, since any evidence and conclusions inferred by AHS-HI Matrix will have high likelihood to be correct (Occam's razor validity) and low likelihood to be incorrect (Chatton's anti-razor validity).

\section{KEY POINTS}

\section{What is Already Known}

- Health depends not only on healthcare system, since decisions made in other sectors impact population health;

- Addressing social determinants of health is important for reducing HI;

- Health in All Policies approach aims to improve population health by taking into account impacts on health from different policies in all sectors;

- HIA can assist decision-makers for making the right choices by predicting the impacts on population health from different policy options;

- There is no gold standard for establishing and assessing validity of predictions in HIA. 


\section{What this Paper Adds}

- This paper makes a distinction between two types of causal pathways regarding health: specific causal pathways and conditional causal pathways;

- It is suggested that HIA should be concerned with establishing and assessing the conditional causal pathways;

- The paper explains the usefulness of AHS-HI Matrix:

- As a gold standard for establishing and assessing all kinds of validity, adding one more - validity of evidence;

- For critical appraisal of the evidence;

- For dealing with complexity and uncertainties;

- For developing and assessing different scenarios of policy options and their impacts on population health;

- For applicability in all sectors, at all levels, and for all kinds of policies.

\section{Policy Implications}

- Sustainable development and population health are intrinsically linked, which requires all policies to be assessed for their health impacts;

- Decision-makers should take into account these impacts and should make choices which improve health of whole populations (i.e. improve AHS while reducing $\mathrm{HI}$ );

- Policies in all sectors should be designed to avoid double losers, a process in which AHS-HI Matrix facilitates making the right choices.

\section{EXAMPLES}

\section{Case I, Fruits and Vegetables (F\&V)}

In 2006 there was a costly advertising campaign, including TV, "2 fruits \& 3 vegies", aiming to improve the daily diet of Australians, most of whom were not consuming enough F\&V. Since January 2007 I give this intervention as example for Case 1 from AHS-HI Matrix (improving AHS and increasing HI). I argue that this campaign, although that might be effective in increasing the consumption of $\mathrm{F} \& \mathrm{~V}$, will result in widening of $\mathrm{HI}$, since the intervention benefits more those individuals who already are better-off (previous winners). Having more disposable income facilitates making healthier choices, while less disposable income can limit these. For example, if one is from a poor family and one learns from this campaign that consuming more $\mathrm{F} \& \mathrm{~V}$ is good for one's health, it will make little difference to one's choices when buying food, since 1 $\mathrm{kg}$ tomatoes cost as much as $1 \mathrm{~kg}$ minced meat ${ }^{1}$ and one knows from one's personal experience that $1 \mathrm{~kg}$ meat will keep one's family fed for longer than $1 \mathrm{~kg}$ tomatoes. In spite of increased awareness about the benefits from consuming more $\mathrm{F} \& \mathrm{~V}$, due to budget constraints one will have to skip the tomatoes, unless there is some kind of incentive like vouchers or subsidy for $\mathrm{F} \& \mathrm{~V}$, for example. This way the new intervention will benefit more those individuals who are worse-off before the intervention (previous losers), the intervention will "work" AHS will improve and $\mathrm{HI}$ will diminish, which will be example for Case 2 from AHS-HI Matrix (improving AHS and decreasing $\mathrm{HI}$ ).

This intervention, which intended to change personal behavior, has led to unintended positive results: (i) governmental businesses and some progressive managers of private businesses 
in good financial situation have started to provide fruits only to their employees at workplace; and (ii) some private businesses have started to provide new service - home delivery of F\&V. Although representing an enhancement of the positive impacts from the initial intervention, this also leads to increasing of $\mathrm{HI}$, since once again those who benefit from it are the previous winners (better-off before), i.e. Case 1 from AHS-HI Matrix (improving AHS and increasing $\mathrm{HI}$ ). Employees in businesses which are cutting costs, part-time and casual workers, as well as sub-contractors do not benefit at all, unless F\&V were tax-deductible, for example, which apparently they are not. Similarly, lower socio-economic groups opt-out home delivery, as they know from their own experience that the extra money for the service would be better spent for more food or something else, unless the service were subsidized for them, for example, which apparently is not.

\section{Case II, Solar Panels (SP)}

With a primary objective reducing greenhouse gas emissions and reducing future investments for production and transmission of electricity the Australian Government provided substantial subsidies of up to AUD 8,000 for households who install SP. The intervention has become so

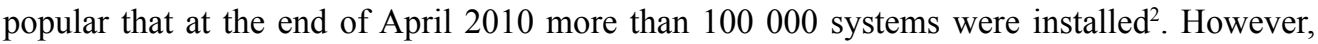
providers of electricity felt the impact, as uptake of SP effectively means decline in demand for electricity and respectively in their profits. This negative impact for the electricity providers was quickly compensated by substantial price increase (unintended negative impact on population). I am not aware if HIA was conducted in regards to this policy, producing recommendations based on analysis of potential impacts and their distribution among the population. Although that the concrete causal pathways regarding health impacts might not be clear or known, this policy is example for Case 5 from AHS-HI Matrix (improving AHS and increasing HI), even if the gain of the winners exceeds the loss of the losers (Kaldor-Hicks Criterion). Subsidising solar panels is a policy which takes from all tax-payers, including the renters (previous losers), and benefits home owners (previous winners). On top of this, previous winners - the home owners - benefit from the change twofold: (i) they get the subsidy for SP; and (ii) they are less exposed to the higher electricity price, as they produce some of the electricity for their home. Previous losers - the renters and people from lower socio-economic groups who opt-out $\mathrm{SP}$ - are absolute losers as they are worse-off compared to their previous situation. They are now twice losers from the new policy: (i) they get no subsidy; and (ii) they are fully exposed to the higher electricity price. The result is growing disparities in disposable income between renters and home-owners. Since the disposable income determines one's choices (see Case I- F\&V), previous losers (the renters) have less ability for making healthy choices, which leads to growing HI. Enhancement of positive impacts from this policy will lead to further increase in HI, unless policy changes are made to benefit specifically the previous losers (the renters).

\section{Case III, Smart Meters (SM)}

Victorian Government started to install free-of-charge new electricity meters - SM - with primary objective to upgrade existing infrastructure in order to keep infrastructure costs down in the future. SM will be installed to 2.5 million homes and businesses at the rate of about 4000 a week, with the promise that these "will actually help reduce the costs of delivering power" according to the website of Department of Primary Industries, Victoria ${ }^{3}$. Ironically (or due to Case II, SP), the price of electricity went up by approx 50\% (Dec 2009 - Dec 2010) and will triple by 2020 according to Grant King, CEO of Origin Energy, who noted "We think that, by

Copyright (C) 2015 The Author. This article published as an Open Access article distributed under the terms of the Creative Commons Attribution NonCommercial 4.0 License (http://creativecommons.org/licenses/by-nc/4.0/). 
2020, the cost of electricity will be threefold what it is today, given the current policy of large amounts of renewables being forced into the system". ${ }^{4}$

Apparently, there is absolutely no benefit from SM for any group in the population. In fact, all groups are worse-off compared to their previous situation as they pay much higher electricity price. Assuming that those who were better-off before the intervention - higher socio-economic groups - have higher electricity consumption than lower socio-economic groups, they would have greater exposure to the higher price, thus will lose more. Although that the concrete causal pathways regarding health impacts might not be clear or known, this intervention is example for Case 8 from AHS-HI Matrix (declining AHS and reducing HI) provided that the loss of the previous winners exceeds the loss of the previous losers. However, considering the above mentioned Case II - SP, previous winners are likely to install SP and reduce their exposure to higher electricity prices, thus compensate their loss, which leads to Case 3 from AHS-HI Matrix (declining AHS and increasing HI). Enhancement of positive impacts which do not target specifically previous losers will lead to further HI increase.

\section{Case IV, Nurse-on-Call (NoC)}

In response to long waiting time in emergency departments in public hospitals Victorian Government introduced new program - Nurse-on-Call - which is "a phone service that provides immediate, expert health advice from a registered nurse, 24 hours a day, 7 days a week", 5 with primary objective to reduce waiting time. This service benefits mainly people from lower socioeconomic groups without private health insurance, who can get expert health advice for the cost of a local cal, i.e. previous losers are now winners from the new intervention. People from higher socio-economic groups with private health insurance (previous winners) visit private hospital, usually with zero waiting time. From this intervention no population group is worse-off compared to their previous situation. Although that the causal pathways regarding health impacts might not be very clear, this intervention is example for Case 2 from AHS-HI Matrix (improving AHS and decreasing HI). Any use of this intervention from people with private health insurance (previous winners), although unintended, represents an enhancement of positive impacts which does not lead to increasing HI.

\section{CONCLUSION}

Economic, social and environmental policies are linked, interact and have impact on population health. No matter how infinitely diverse populations and local contexts can be, there always will be winners and losers from different policy options, and their specific combinations will determine their impact on AHS and HI. By taking into account these impacts Health in All Policies can improve efficiency in relation to health, which is ethical, as it helps achieving the overarching goal for improving health of whole populations. In order to be successful Health in All Policies needs reliable predictions about impacts on population health from different policy options. However, without a gold standard predictions can be problematic. AHS-HI Matrix explains the generative mechanisms which shape population health. Since the matrix always identifies the true condition and provides universal explanations and predictions regarding impacts on population health from different policy options in terms of changes in AHS and HI, AHS-HI Matrix can be used as a gold standard for establishing and assessing validity of predictions. The matrix meets all forms of validity and is particularly useful for solving problems with the evidence. AHS-HI Matrix facilitates making the right choices at any level and in any local context, thus is a useful tool for researchers, policy-makers and practitioners for designing, analysing and/or evaluating all kinds of policies, projects, programs or interventions on populations. By allowing swift, reliable and

Copyright (C) 2015 The Author. This article published as an Open Access article distributed under the terms of the Creative Commons Attribution NonCommercial 4.0 License (http://creativecommons.org/licenses/by-nc/4.0/). 
inexpensive appraisal of different policy options the matrix makes taking into account impacts on population health feasible and paves the way for institutionalizing of Health in All Policies.

\section{REFERENCES}

Bamberger, M., \& Segone, M. (2011). How to design and manage Equity-focused evaluations (p. 9). UNICEF Evaluation office, New York.

Banken, R. (1999). From concept to practice: Including the social determinants of health in environmental assessments. Canadian Journal of Public Health, 90(Suppl. 1), S27-S30. PMID:10686756

Barrow, C. (2000). Social Impact Assessment: an introduction. London: Edward Arnold.

Becker, H. (1997). Social impact assessment: method and experience in Europe, North America and the Developing World. Social Research Today (Vol. 10). London: UCL Press.

Connelly, J. (2001). Critical realism and health promotion: effective practice needs an effective theory [Editorial]. Health Edu Research, 16(2), 115-120. Oxford University Press

Connelly, J. (2005). More public health theory please - but make it adequate [Editorial]. Journal of Public Health, 27(4), 315. doi:10.1093/pubmed/fdi061 PMID:16234260

Connelly, J. (2007). Evaluating complex public health interventions: Theory, methods and scope of realist enquiry. Journal of Evaluation in Clinical Practice, 13(6), 935-941. doi:10.1111/j.1365-2753.2006.00790.x PMID:18070265

Douglas, M., \& Scott-Samuel, A. (2001). Addressing health inequalities in health impact assessment. Journal of Epidemiology and Community Health, 55(7), 450-451. doi:10.1136/jech.55.7.450 PMID:11413171

Heller, R., Weller, D., \& Jamrozik, K. (2004). UK health inequalities: The class system is alive and well. MJA, 181(3), 128. PMID:15287828

Kauppinen, T., Nelimarkka, K., \& Perttila, K. (2006). A case study of the role of health impact assessment in implementing welfare strategy at local level. Health in All Policies - Prospects and Potentials (Ch. 13). Ministry of Social Affairs and Health, Finland.

Kelly, M., Bonnefoy, J., Morgan, A., \& Florenzano, F. (2006). The Development of the evidence base about the social determinants of health. WHO. Retrieved from http://www.who.int/social_determinants/ resources/mekn_paper.pdf

Kemm, J. (2006). Health impact assessment and Health in All Policies. Health in All Policies - Prospects and Potentials (Ch. 10). Ministry of Social Affairs and Health, Finland

Lehto, J., \& Ritsatakis, A. (1999, October 28-31). Health impact assessment as a tool for intersectoral health policy (Discussion paper). Proceedings of WHO conference -HIA: From Theory to Practice, Gothenburg.

Marmot, M., Friel, S., Bell, R., Houweling, T., \& Taylor, S. (2008). Closing the gap in a generation: health equity through action on the social determinants of health. WHO, Geneva. Retrieved from www.who.int/ social_determinants/final_report/en

Melkas, T. (2013). Health in all policies as a priority in Finnish health policy: A case study on national health policy development. Scandinavian Journal of Public Health, 41(Suppl. 11), 3-28. doi:10.1177/1403494812472296 PMID:23434760

Mindell, J., Boaz, A., Joffe, M., Curtis, S., \& Birley, M. (2004). Enhancing the evidence base for health impact assessment. Journal of Epidemiology and Community Health, 58(7), 546-551. doi:10.1136/ jech.2003.012401 PMID:15194713

Panayotov, J. (2006a). Child Health - Increasing Average Health Status or Decreasing Health Inequalities. In touch, Newsletter of the Public Health Association of Australia, 23(2). Retrieved from http://www.phaa. net.au/documents/intouch_mar06.pdf 
Panayotov, J. (2006b, August). Equity -A Premise for Efficiency in Public Health. Paper presented at 11th World Congress on Public Health, Rio de Janeiro, Brazil. Retrieved from http:/icare.academia.edu/JordanPanayotov/Talks

Panayotov, J. (2008a, August 8). Public Health and Average Health Status: Do Health Inequalities Matter? ICARE. Retrieved from http://icare.academia.edu/JordanPanayotov/Papers

Panayotov, J. (2008b, October). Health Impact Assessment: Is Sustainable Wellbeing Possible Without Health Equity? Paper presented at 9th International HIA Conference, Liverpool, UK. Retrieved from http:// icare.academia.edu/JordanPanayotov/Talks

Panayotov, J. (2009, February 1). Evidence in Public Health and Health Impact Assessment. ICARE. Retrieved from http://icare.academia.edu/JordanPanayotov/Papers

Panayotov, J. (2010, April 7). What should we strive for in urban development - increasing Average Health Status or decreasing Health Inequalities: the role of HIA. Paper presented at WHO Health Impact Assessment Conference, Geneva, Switzerland. Retrieved from http://icare.academia.edu/JordanPanayotov/Talks

Panayotov, J. (2011). Equity Focussed Health Impact Assessment - A Step Forward or Two Steps Backwards. ICARE. Retrieved from http://icare.academia.edu/JordanPanayotov/Papers

Popper, K. (1957). The poverty of historicism (2nd ed., pp. 130-143). London: Routledge \& Kegan Paul.

Thérivel, R., Wilson, E., Thompson, S., Heany, D., \& Pritchard, D. (1992). Strategic Environmental Assessment. London: Earthscan Publications Ltd.

Vanclay, F., Esteves, A. M., Aucamp, I., \& Franks, D. (2015). Social Impact Assessment: Guidance for assessing and managing the social impacts of projects. Fargo, ND: IAIA.

Veerman, L., Mackenbach, J., \& Barendregt, J. (2007). Validity of predictions in health impact assessment. Journal of Epidemiology and Community Health, 61(4), 362-366. doi:10.1136/jech.2006.047480 PMID:17372299

Venkatapuram, S. (2010). Global Justice and the Social Determinants of Health. Ethics \& International Affairs, 24(2), 119-130. Retrieved from https://www.carnegiecouncil.org/resources/journal/24_2/essays/001 doi:10.1111/j.1747-7093.2010.00252.x

Vitanyi, P., \& Li, M. (2000). Minimum Description Length Induction, Bayesianism, and Kolmogorov Complexity. IEEE Transactions on Information Theory, 46(2), 446-464. doi:10.1109/18.825807

WHO. (2014). HiAP Framework for Country Action. Geneva: WHO.

\section{ENDNOTES}

12007 prices: $1 \mathrm{~kg}$ truss tomatoes $\$ 6.99 ; 1 \mathrm{~kg}$ minced regular beef on special $\$ 6.99$, July 2013 prices: $1 \mathrm{~kg}$ truss tomatoes $\$ 6.98 ; 1 \mathrm{~kg}$ minced regular beef every-day $\$ 5.00$

2 Department of Climate Change \& Energy Efficiency, Australian Government, "Solar Homes \& Communities Plan", retrieved January 12, 2011, from http://www.climatechange.gov.au/what-youneed-to-know/renewable-energy/solar-homes.aspx

3 Department of Primary Industries, Victoria, “Why Smart Meters?", retrieved January 12, 2011, from http://new.dpi.vic.gov.au/smart-meters/why-smart-meters/benefits

4 The Australian, "Energy prices to triple, says Origin chief", April 14, 2010, retrieved January 12, 2011, from http://www.theaustralian.com.au/business/energy-prices-to-triple-says-origin-chief/ story-e6frg8zx-1225853385647

5 Nurse-on-Call, Department of Health, Victoria, retrieved January 12, 2011, from http://www.health. vic.gov.au/nurseoncall/about.htm 